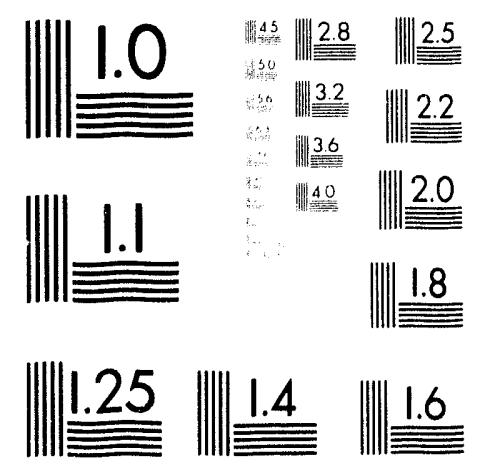



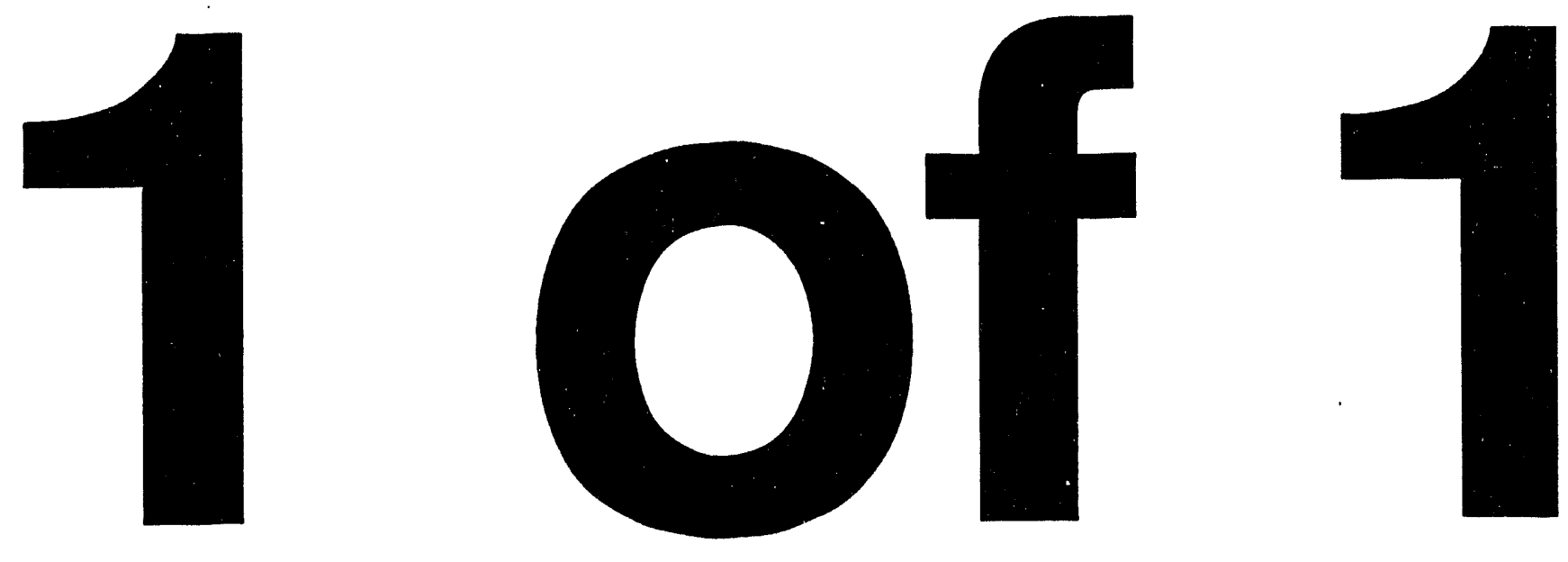
SC-MAG-417

LBL-33439

\title{
Evaluation of APC NbTi Superconductor in a Model Dipole Magnet*
}

\author{
R. M. Scanlan, A. Lietzke, J. Royet, \\ A. Wandesforde, and C. E. Taylor \\ Accelerator and Fusion Research Division \\ Lawrence Berkeley Laboratory \\ University of Callfornia \\ Berkeley, California \\ J. Wong and M. K. Rudziak \\ Supercon, Inc. \\ Shrewsbury, Massachusetts
}

September 20, 1993

\footnotetext{
* This was supported by the Director, Office of Energy Research, Office of High Energy and Nuclear Physics, High Energy Physics Division, U. S. Department of Energy, under Contract No. DE-AC03-76SF00098. 


\title{
Evaluation of APC NbTi Superconductor in a Model Dipole Magnet
}

\author{
R.M. Scanlan, A. Lietzke, J. Royet, \\ A. Wandesforde, and C. E. Taylor \\ University of California, Lawrence Berkeley Laboratory, \\ Berkeley, CA 94720 \\ J. Wong and M.K. Rudziak \\ Supercon, Inc. \\ Shrewsbury, MA 01545
}

\begin{abstract}
The artificlal plnning center (APC) approach to NbTi superconductor tabrication offers the potentlal beneflts of higher current density and lower cost than the conventlonal process for NbTl. We have been evaluatlng several approaches for fabricating NbTI via the APC approach to determine whether these advantages can be reallzed in a practical conductor. The study began with the fabrication by several vendors of $10 \mathrm{~kg}$ size samples whlch were evaluated as short samples. This was followed by the scale-up of one process to $150 \mathrm{~mm}$ diameter billets. This material was evaluated first in a solenold configuration and recently in a one-meter long dipole. We will report bere on the results of these coll tests and other characterization results for thls new material. We will also describe the plans to continue the scale-up to full slze blllets and we will discuss the potential cost savings of this approach compared with conventional NbTi fabrlcation.
\end{abstract}

\section{INTRODUCTION}

During the last 10 years, significant improvements have been made in understanding and controlling the factors which affect the critical current density in conventional $\mathrm{NbTi}$ superconductors. This has led to a $50 \%$ improvement in the critical current density available to magnet designers for the SSC magnets compared to that for the Tevatron magnets [1]-[2]. Laboratory experiments on samples of NbTi with very well aligned pinning centers have shown that the potential still exists for further improvement to current densities above $5000 \mathrm{~A} / \mathrm{mm} 2$ at $5 \mathrm{~T}$ [3]. However, these improvements have not been transitioned from the laboratory to practical conductors. The primary difficulties that have prevented this transition are the following: In order to produce the high volume fraction of precipitates required for optimum flux pinning, many long duration, high temperature heat treatments are needed. These beat treatments cause a general deterioration of the filament quality due to interdiffusion with the $\mathrm{Cu}$ matrix and also to the softening of the matrix relative to the filaments. Also, the size and alignment of the flux line pinning precipitates cannot be optimized.

This work was supporied by the Director, Office of Energy Research, Orfice of High Energy and Nuclear Physics. High Energy Physics Division, U.S. Department of Energy, under Contract No. DE-AC03765 F00098.
This paper describes the application of a new fabrication approach, called artificial pinning centers (APC), which offers the promise of overcoming the limitations of the conventional process described above, and also promises to be significantly less costly than the conventional process NbTi. We will describe this process and the status of the effort to scale up the process to commercial size billets. We will also report on the results of magnets made from this new material and compare the performance with the conventional NbTi superconductors.

\section{A. APC conductor process for $\mathrm{NbTi}$}

- The basic idea behind the APC approach for NbTi is to replace the alpha- $T i$ precipitate pinning centers with "artificial" pinning centers, whose composition, size, and spacing can be changed and optimized easier than those properties of the alpha-Ti precipitates. The optimum diameter for the pinning ceriters is roughly twice the coherence length, or $11 \mathrm{~nm}$. The optimum spacing would correspond to the flux line spacing at the field of interest, e.g., $25 \mathrm{~nm}$ for 5T. The first published attempt to apply the APC concept to NbTi was reported at MT-9 by workers from the Kurchatov Institute [4]. They reported a maximum Jc (5T, 4.2K) of $3500 \mathrm{~A} / \mathrm{mm} 2$ in a wire made with a Nb-50 wt\% Ti matrix and $\mathrm{Nb}$ pinning centers which were introduced as microfilaments in the NbTi matrix. The optimum Jc was achieved when the $\mathrm{Nb}$ microfilament diameters and their spacing was about 50nm. Attempts to increase Jc by further refining the structure were not successful, due to "mechanical and diffusional destruction of the APC" [4]. More recently, Kanithi [5], reported achieving similar Jc values for a NbTi APC by a different process, in which the NbTi filaments are clad with $\mathrm{Nb}$ and bundled together to produce a filament. Encouraged by these promising results, our group at LBL solicited proposals from wire manufacturers on different methods for fabricating APC type structures. Intermagnetics General Corp. (IGC) proposed to scale-up the NbTi core/Nb clad process; Teledyne Wah Chang Albany (IWCA) proposed to explore a jelly roll approach with alternating wraps of $\mathrm{NbTi}$ and $\mathrm{Nb}$ sheets; and Supercon proposed a very novel approach using pure $\mathrm{Nb}$ and pure $\mathrm{Ti}$ sheets wrapped into a jelly roll configuration [6]. At the end of the initial evaluation phase, we decided to pursue scale-up of the Supercon process, sinct it showed the following advantages. First, the multifilamentary composites produced by their process showed excellent critical current properties, both at $5 \mathrm{~T}$ and also at higher fields. For example, wire has a JC $(5 \mathrm{~T}, 4.2 \mathrm{~K})=3340 \mathrm{~A} / \mathrm{mm} 2$ and a Jc $(T \mathrm{~T}, 4.2 \mathrm{~K})=1615$ 
$\mathrm{A} / \mathrm{mm} 2 . ;$ an optimized wire made by the IGC process has a comparable Jc $(5 \mathrm{~T}, 4.2 \mathrm{~K})=3370 \mathrm{~A} / \mathrm{mm} 2$, but a Jc $(7 \mathrm{~T}$. $4.2 \mathrm{~K}$ ) of only $1020 \mathrm{~A} / \mathrm{mm} 2$ (Fig. 1.) There are some basic limitations in the type of process being pursued by IGC and TWCA which account for this difference in high field performance, which are discussed below. Second, the Supercon process has some inherent cost advantages over the processes which use NbTi alloy sheet. The cost of pure metal $\mathrm{Nb}$ and $\mathrm{Ti}$ sheets, projected to production scale quantities, will be considerably lower than the cost of $\mathrm{NbTi}$ alloy sheet. The projected cost savings for the APC superconductor, both in terms of raw materials and processing costs, will be discussed later in more detail.

A step by step description of the Supercon process is shown in Fig. 2. In the first step, an analog to the conventional process monofilament is prepared from an assembly of pure $\mathrm{Nb}$ and pure $\mathrm{Ti}$ sheets. These sheets can be assembled as a jelly roll, as shown in Fig. 2. After stacking, the sheets are surrounded by a pure niobium diffusion barrier and inserted into a copper can, just as for conventional process monofilaments. The billet is then electron beam welded, hot or cold isostatic pressed, and extruded to produce the copper clad monofilament. This monofilament is then drawn to the appropriate size and hexed in preparation for stacking in the multifilament billet. The rods are cut to length, cleaned, and stacked in a copper can. The can is closed by welding, cold or hot isostatically pressed, and then extruded. Up to this point, the processing for the APC billet is the same as for a conventional NbTi billet except that the monofilament billet is made of stacked sheets instead of a $\mathrm{NbTi}$ alloy ingoc.

After the multifilamentary extrusion step has been completed, the processing for the APC billet is much easier and faster. Whereas the conventional material must undergo a series of drawing and heat treatment operations, the APC material can be cold drawn without intermption to the final wire size. The annealing sequence for the conventional $\mathrm{NbTi}$ has three drawbacks. Firsh the drawing process must be interrupted and the wire, coiled for heat treatment. In some instances the wire must be cleaned and/or coiled on speciai spools for this heat treatment. The heat treatments are very long, typically $80 \mathrm{hr}$ at $375 \mathrm{C}$ or $40 \mathrm{hr}$ at $425 \mathrm{C}$. This heat treatment fully anneals the $\mathrm{Cu}$ matrix and thus produces a large difference in mechanical properties between the matrix and the filaments, which can cause filament sausaging during subsequent drawing. Also, interdiffusion at the filament boundary occurs, and often the duration and/or temperature of the heat treatment must be reduced below that which would give optimum critical current performance in order to prevent filament sausaging or wire breakage. These heat treatment steps are most critical for obtaining high Jc values and good piece lengths. Much of the technology development for conventional $\mathrm{NbTi}$ has focused on the optimization of these heat treatment steps, and some of the details, such as optimum extrusion temperature, beat treatment time/temperature, and reduction in area between heat treatments, still must be determined empirically for each lot of NbTi alloy from different sources.
Thus, the intermediate heat treatments add much to the cost and complexity of processing conventional $\mathrm{NbTi}$. These costs will be discussed more in a later section.

\section{B. Process optimization with small billets}

Process optimization for conventional NbTi is difficult to perform on small size billets because a minimum amount of cold drawing must be performed between each beat treatment step in order to achieve high $\mathrm{Jc}$ values (this is often referred to as the minimum strain space limitation [7]). In the case of the APC process, fully optimized critical current densities can be achieved in $51 \mathrm{~mm}$ diameter billets. Hence, only small amounts of material must be expencied in this process so that optimization costs and time are reduced.

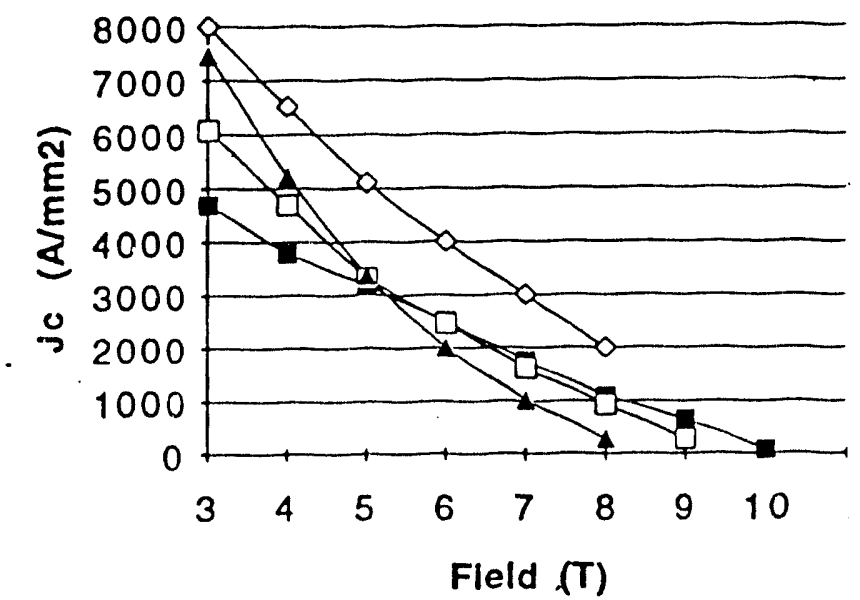

Fig. 1. Comparison of JC vs. field behaviox for wires made by the IGC APC process (solid triangle). Supercon APC process (open square), ind conventional $\mathrm{NbTi}$ process (solid square). Also included is the best laboralory sample dula on a flatlened ribbon sample (open diamood [3]).

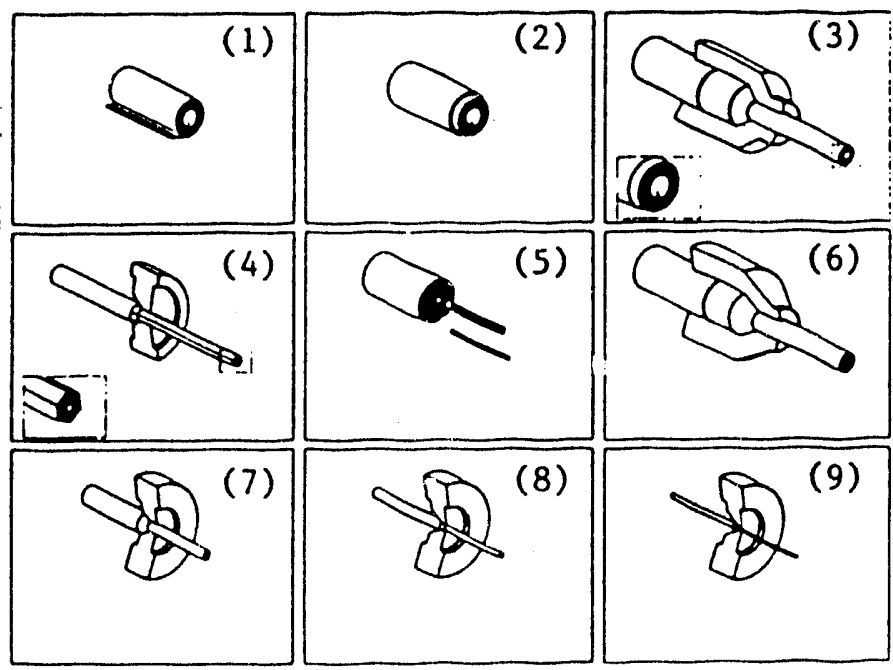

Fig. 2. Sequence of manufacturing steps for Supercon APC process. (1) wrap alternating $\mathrm{Nb}$ and $\mathrm{Ti}$ sheets, (2) load into a Cu can, (3) extrude monofilament, (4) draw, hex and cut to stacking length, (5) load into mulufilamentary billet, $(6)$ extrude mulufilamentary billet, $(7,8,9)$ draw to final wire size. 
One of the more important conclusions from these optimization studies is that the Supercon APC process allows the critical current to be selectively optimized at different fields and at different wire sizes. For example, the critical current density vs. field is plotted for two different wires produced from $51 \mathrm{~mm}$ diam. billets in Fig. 3. This effect is also evident in the comparison of Jc vs. field curves for the APC wires used in the inner and outer layer cables in the D19B dipole magnet, discussed later. After the initial process optimization studies with $51 \mathrm{~mm}$ billets were complete, Supercon was authorized to begin scale-up studies aimed at producing $130-150 \mathrm{~mm}$ diameter billets.

\section{Scale-up to intermediate size billets}

During this phase, the following questions were addressed: (1) can properties equivalent to those achieved in $51 \mathrm{~mm}$ billets be achieved in 130-150mm diam. billets? (2) can the APC material be produced in long lengths? (3) do magnets produced with the APC material perform in the same way as magnets from conventional material? The type of magnet chosen for this evaluation is a solenoid which will achieve at least $8 \mathrm{~T}$ in a useful clear bore and that can be operated in a persistent current mode. This type of magnet iest answers many of the important questions concerning long piece length, uniform properties, and high field performance.

The monofilament billet consisted of a jelly roll configuration of $\mathrm{Nb}$ and $\mathrm{Ti}$ sheets wrapped around a NbTi core and loaded in a $150 \mathrm{~mm}$ diam. Cu can. The billet was sealed, compacted and extruded. The extruded rod was drawn to $0.76 \mathrm{~mm}$ diam., cut into lengths, and reloaded in a $128 \mathrm{~mm}$ diam. billet. This billet was sealed, extruded, and drawn to $1.14 \mathrm{~mm}$ diam. wire containing 2994 filaments. The wire was insulated with Formvar and sent to LBL where it was wound into a solenoid magnet. Details of the coil winding and magnet testing have been described in an earlier paper [8].

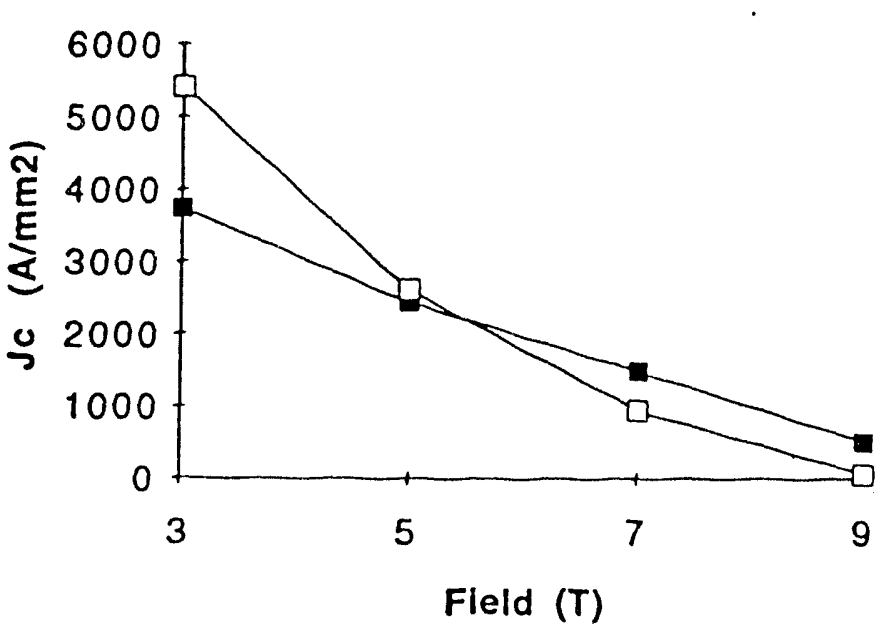

Fig. 3. Effect of processing conditions on properies of APC wire. Results are for same starting monofilament and same final wire size.
The expected magnet performance from short sample tests is compared with the actual magnet performance in Fig. 4. The magnet achieved a central field of $8.5 \mathrm{~T}$ and operated surcessfully at the design field of $8.0 \mathrm{~T}$ in the persistent current mode. Since the initial tests were completed, this magnet has been used to provide the background field for short sample critical current tests for the past two years, without any problems. Thus, this magnet has successfully demonstrated the high field performance, uniform properties in long piece lengths, and the production of long, continuous pieces which were the main goals of this phase of the scaleup program.

\section{Scale-up to SSC-type wire}

The next phase of the scale-up was the fabrication of SSCtype wire, i.e. wire with 6-8 micron diameter filaments in the standard wire sizes used in the inner and outer layer cables for SSC dipoles. The primary goal of this phase of the scale-up program was to produce enough wire so that an accelerator type dipole magnet could be constructed. The monofilament billet diameter was beld at $150 \mathrm{~mm}$, since at this size only two monofilament and two multifilamentary billets are required to produce the wire necessary for a $1 \mathrm{~m}$ long dipole. The monofilament billets were assembled in the jelly roll configuration used in the earlier development phase.

The monofilaments were extruded and then part of the material was used to prepare a number of $50 \mathrm{~mm}$ diam. secondary extrusions which were processed under different conditions in order to optimize the processing. This optimization study showed that a Jc (5.6T, 4.2K) of 2650 A/mm 2 could be achieved, which is substantially above the SSC specification value of $2440 \mathrm{~A} / \mathrm{mm} 2$. A total of 6 billets, each $50 \mathrm{~mm}$ in diam., were extruded and processed to produce the wire for the outer layer cable.

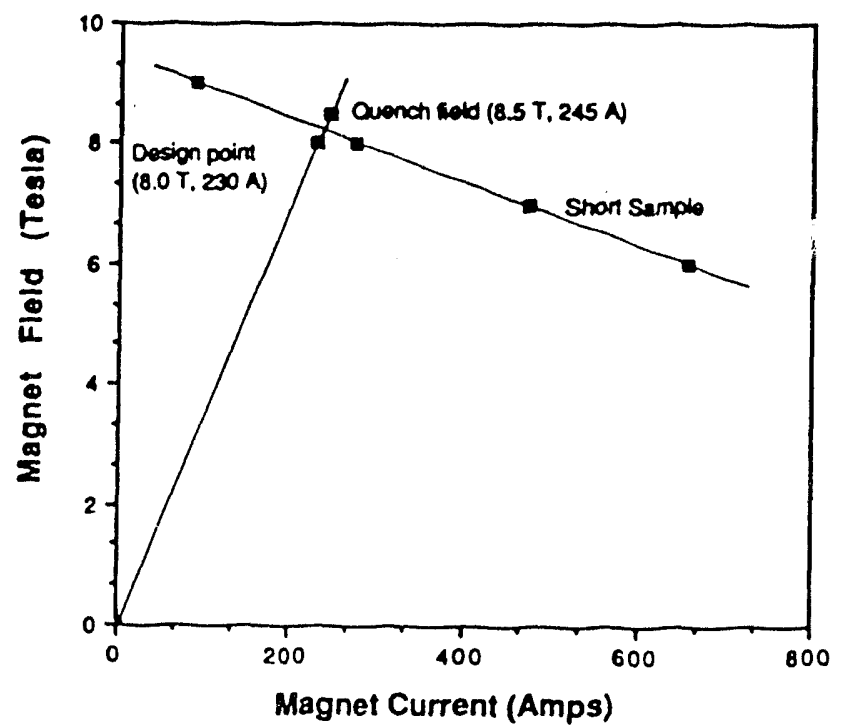

Fig. 4 Solenoid magnet load line and wire short sample critical curreat line. The maga et reaclied a field of $8.5 T$ at $245 \mathrm{~A}$, which is slightly above the short sample critical current value. 
The properies of this wire, compared to the SSC wire specifications, are shown in Table 1. The average Jc (5.6T, $4.2 \mathrm{~K}$ ) value is about $10 \%$ lower than the SSC spec. value. The reason for the drop in Jc from the initial optimization studies to the production series is not completely clear. Image analysis performed on these wires indicate that the degree of filament sausaging, expressed as the ratio of the standard deviation to average value, is $12.5 \%$ compared to a typical value of about $6 \%$ for recent SSC wire [9]. This sausaging may be the result of a slightly higher ratio of spacing to diameter $(0.21$ instead of the specifiet value of 0.17).

The inner layer wire was produced from a scaled-up secondary billet, $130 \mathrm{~mm}$ in diam. The inner layer multifilament billet was extruded and cropped for inspection. This inspection showed that the billet had upset more than expected during the extrusion step, as seen in Fig. 5. This upset was due to the increased void space in this billet due to the large number of elements (over 6000) and the fact that the billet was not cold or hot isostatically pressed. Initial metallography showed filaments as targe as $16 \mu \mathrm{m}$; however. subsequent cross sections of 30 strands in a cable showed some regions of more severe distortions where the filament diameter was as large as $50 \mu \mathrm{m}$.

Past experience with conventional NbTi composites has shown that wire with this degree of filament distortion would experience severe breakage during wire drawing. Surprisingly, this APC material was drawn to final size in one single piece $3300 \mathrm{~m}$ long, without any breaks. This result is further proof that the elimination of the multiple heat treatments associated with the conventional NbTi processing greatly improves fabricability and piece length. In order to improve the critical current of the inner layer wire, the copper to superoonductor was reduced from the nominal 1.3:1 to about $0.9: 1$ by etching away some of the copper matrix during processing. The values of $\mathrm{IC}$ and $\mathrm{JC}$ for this wire are listed in Table 1. Notwithstanding the rather severe distortion of the filaments, the Jc value at $7 \mathrm{~T}$ is within $15 \%$ of the value specified for SSC type wire, and the n-value is above 30 .

TABLE 1

PROPERTIES OF SUPERCON APC WIRE USED IN DIPOLE DI9B

\begin{tabular}{|c|c|c|c|c|c|c|c|}
\hline Type & Qu:SC & $\begin{array}{l}\mathrm{Jc}(5.6 \mathrm{~T}) \\
(\mathrm{A} / \mathrm{mm} 2)\end{array}$ & $\begin{array}{c}\operatorname{Ic}(5.6 \mathrm{~T}) \\
(\mathrm{A})\end{array}$ & $\mathrm{Jc}(\mathrm{TT})$ & $\operatorname{lc}(7 T)$ & $\operatorname{Jc}(9 T)$ & $\operatorname{Ic}(9 T)$ \\
\hline $\begin{array}{l}\text { Inger } \\
.81 \mathrm{~mm}\end{array}$ & $.85: 1$ & & & $\begin{array}{l}\text { 140S(ave) } \\
\text { (Range } \\
1374-1437 \text { ) }\end{array}$ & 389 & 278 & 77 \\
\hline $\begin{array}{l}\text { Outer, } \\
.65 \mathrm{~mm}\end{array}$ & $1.4: 1$ & $\begin{array}{l}2200 \text { (ave) } \\
\text { (Range } \\
2003-2334)\end{array}$ & 290 & 806 & 109 & & \\
\hline
\end{tabular}

Nominal filameat size -8 inicrons

$\begin{array}{llllll}\text { SSC } & 1.3: 1 & & & 1650 & 357 \\ \text { Inner } & & & & & \\ \text { Spec } & & & & & \\ \text { SSC } & 1.8: 1 & 2440 & 285 & & \\ \text { Ouler } & & & & & \\ \text { Spec } & & & & & \end{array}$
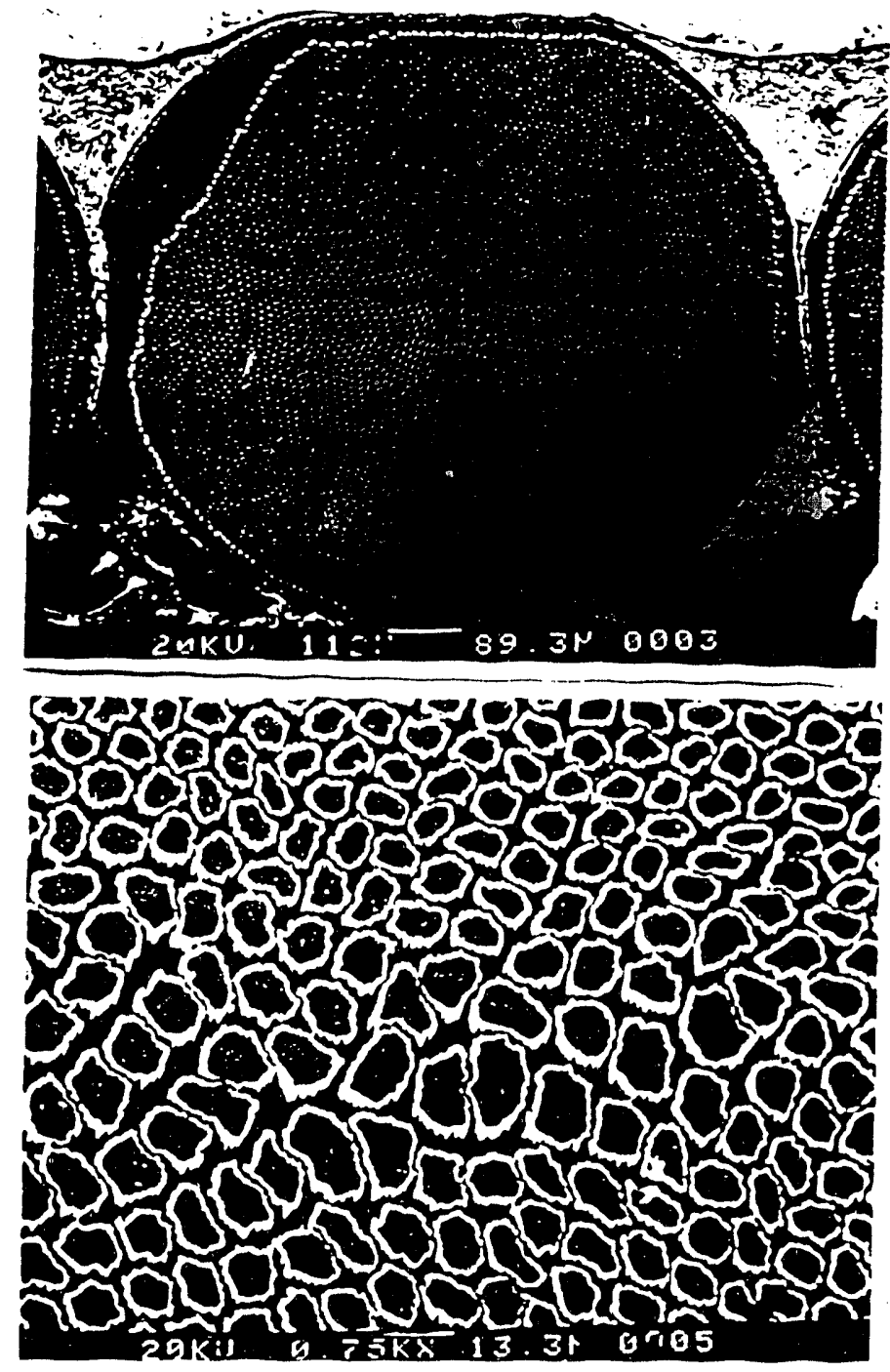

Fig. 5. Transverse sections of the Supercon APC multifilamentary wire used in dipole DI9B. Note that the billet has upset due to excess void apace in the billet at the time of extrusion.

\section{E. Cable fabrication and testing}

This APC material was evaluated in a $1 \mathrm{~m}$ dipole at LBL. The dipole design had previously been developed and tested using SSC-type cables made from conventionally processed NbTi superconductor [10]. The inner and outer cables for this new dipole, called D19B, were fabricated to SSC specifications without difficulty. Critical current measurements were made at BNL, and both cables showed little degradation in critical current due to cabling. The degradation for the inner cable was $2 \%$, and the degradation for the outer cable was $5 \%$, where the degradation is defined as the ratio of the measured cable critical current, corrected for the self field effects, to the value of a single strand multiplied by the number of strands [11]. In addition, a short sample training test was made on the inner cable, and it performed well for a cable with this low $\mathrm{Cu} / \mathrm{SC}$ ratio value of $0.9 / 1$ [12]. The cable n-values of about 30 for boun cables was also quite good, compared to $\mathrm{n}$-values for conventional $\mathrm{NbTi}$ cables. 


\section{F. Dipole magnet construction and testing}

The design chosen for D19B was the same as that used for D19A [10]. D19A utilized a thin stainless steel collar and a close-in elliptical iron yoke in order to obtain the maximum dipole field. This design used the same cable as the $50 \mathrm{~mm}$ bore SSC dipoles, and the design features described above resulted in the achievement of a record field of $10.06 \mathrm{~T}$.

Construction of DI9B using the APC NbTi cables proceeded without incident and the magnet was tested during February/March 1993. The magnet started training at 6.4T and trained up to a plateau field value of $7.0 \mathrm{~T}$ after 6 training quenches (Fig. 6). After cycling to lower temperatures and higher fields, the plateau field reached $7.2 \mathrm{~T}$, which is about 97\% of the value predicted from the wire and cable short sample measurements. We consider this amount of training is relatively good, especially since the inner layer $\mathrm{Cu} / \mathrm{SC}$. ratio is quite low compared with D19A and other SSC type dipoles. The magnet was also trained at $1.8 \mathrm{~K}$ and reached a field of $8.7 \mathrm{~T}$; we do not have the wire test data necessary (tests at $1.8 \mathrm{~K}$ and bigh fields) to construct an accurate critical current line to compare with the magnet performance at $1.8 \mathrm{~K}$. However, the ratio of $\mathrm{R}(1.8 \mathrm{~K}) / \mathrm{B}(4.2 \mathrm{~K})$ is lower for D19B than D19A. Critical current measurements at $1.8 \mathrm{~K}$, to high fields, are in progress.

Magnetization and multipole measurements were also made on D19B and compared with similar results for D19A.

In summary, the performance of the APC NbTi material in this dipole test has demonstrated that its behavior is qualitatively similar to that of the conventional NbTi. Two main problems or differences were identified. First, the billet upset and filament distortion seen in the APC composites are due to well known extrinsic effects, i.e. lack of billet compaction and large filament spacing. These problems are corrected in the composites presently under evaluation. The other difference noted was the slightly lower Jc in the production lot of material compared to the optimization experiment samples. This difference may be due to either intrinsic effects associated with differences in the heat treatments or extrinsic effects due to increased filament sausaging in the production lot. The cause is under investigation in the present series of billets.

\section{G. Recent results and billets in progress}

The next stage of scale-up is now in progress. The goals of this stage are to demonstrate the scale-up to full size production billets and to optimize the Jc in SSC-type wires. First, monofilament billets will be extruded. Pan of the material will be used in optimization studies aimed at selecting the best processing conditions. Then, multifilamentary billets $300 \mathrm{~mm}$ in diameter will be extruded and drawn into wire. This wire will be cabled and then evaluated in another test dipole magnet.

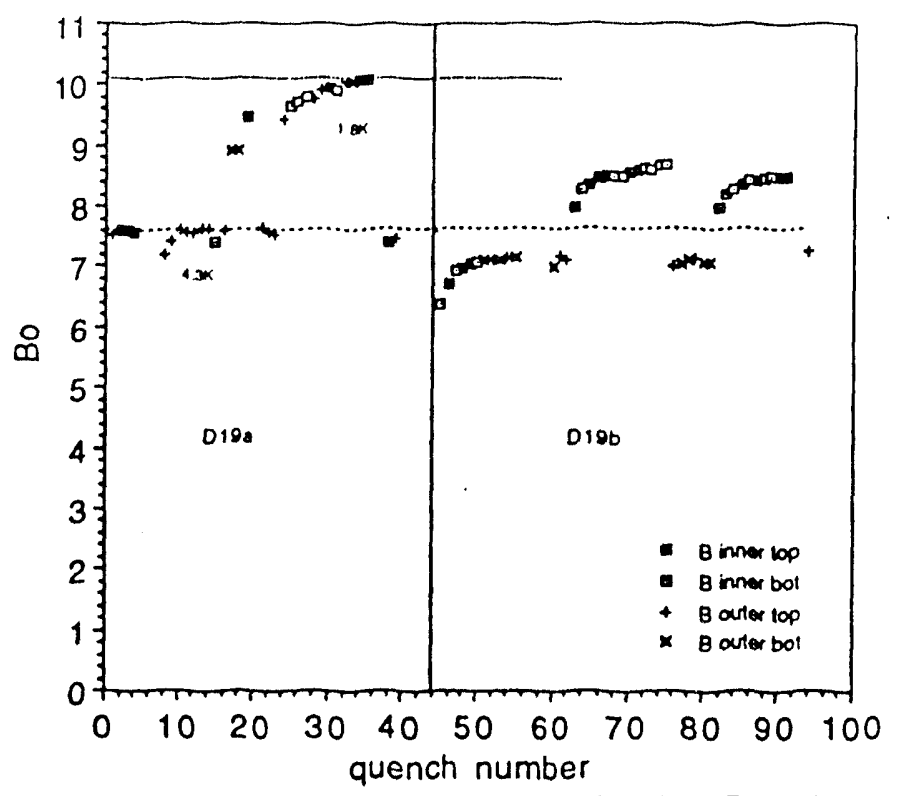

Fig. 6 magnet training curves for D19A (best LBL dipole) and D19B (first dipole with APC material.). D19B resched a platesus field 97\% of the cable shor sample value.

\section{H. Cost implications for APC process NbTi}

Detailed comparison of the costs of APC NbTi compared with conventional process NbTi must await results of the scale-up to full production size multifilamentary billets. However, the preliminary cost comparison results look quite favorable for the APC material. We will first discuss the raw materials cost savings and then the processing cost savings.

Raw Materials. NbTi ingot costs for large quantity purchases such as $S S C$ are $\$ 106 / \mathrm{kg}$. This is a significant fraction of the total costs for conventional NbTi wire; for example, it accounts for 35\% of total costs for SSC inner layer wire [13]. The costs of raw materials for the Supercon APC process are reduced very significantly - the Ti sheet cost is $\$ 22 / \mathrm{kg}$, and the cost of $\mathrm{Nb}$ sheet, projected to large scale quantities, is $\$ 88 / \mathrm{kg}$. Using these values, the comparable costs for raw materials for the APC process is $\$ 55 / \mathrm{kg}$, compared with $\$ 106 / \mathrm{kg}$ for the conventional NbTi.

The costs for $\mathrm{Nb}$ sheet must be projections at this time, since $\mathrm{Nb}$ sheet is not currently being produced in large quantities. As a benchmark, we have a quote for $45 \mathrm{~kg}$ of $\mathrm{Nb}$ sheet $0.5 \mathrm{~mm}$ thick, for $\$ 111 / \mathrm{kg}$. The difference between this quotation and our projection is a question of demand, not technological difficulty. The production of $\mathrm{Nb}$ sheet from electron beam melted $\mathrm{Nb}$ is a standard forging and cold rolling process [14]. The cost of electron beam melted $\mathrm{Nb}$ ingot, which is the starting material for the $\mathrm{NbTi}$ ingot and for $\mathrm{Nb}$ sheet, is $\$ 59 / \mathrm{kg}$. The cost of converting the $\mathrm{Nb}$ to sheet is not so costly as converting NbTi to small diameter rods or sheet, because the $\mathrm{Nb}$ scrap can be recycled, whereas the NbTi scrap cannot. Hence, the allowance of $\$ 29 / \mathrm{kg}$ for processing the $\mathrm{Nb}$ to sheet and recycling costs for the $\mathrm{Nb}$ scrap is quite generous. 
Fabrication costs. Fabrication costs of the APC NbTi also compare quite favorably with the cost of conventional $\mathrm{NbTi}$ fabrication. The only additional cost is that for preparing the jelly roll of $\mathrm{Nb}$ and $\mathrm{Ti}$ sheets. The labor associated with preparing the monofilament billets is only $2 \%$ of the total labor costs for SSC-type conductor, so even if assembly time is doubled, this is an insignificant increase in total labor costs. On the other hand, the APC approach eliminates the intermediate size heat treatments, which accounts for a significant fraction of direct as well as indirect costs of the conventional NbTi conductor. Direct costs are the labor required to (1) coil or respool the rods or wire in preparation for heat treatment, (2) in some cases, remove the lubricant, (3) load the furnaces, (4) monitor the heat treatments, (5) remove the rods or wire from the furnace, and (6) reload the rod or wire drawing machines. Other direct costs include the capital equipment and operating costs of the large furnaces required for these batch anneals. Indirect costs include additional wire breakage associated with these anneals, complex scheduling and material management, and an increase in the time the material is in process, after the multifilamentary extrusion is complete, of at least $100 \%$.

Based on these factors, we estimate that the fabrication cost should be reduced by $40 \%$. Combining the raw materials cost savings with the fabrication cost savings yields a total cost for APC NbTi which is about 55\% the cost of conventional NbTi.

\section{Summary}

1. APC process NbTi superconductors have been optimized to give Jc values at bigh fields which are comparable to those obtained in conventional $\mathrm{NbTi}$.

2. The Supercon process for fabricating APC NbTi has been scaled-up successfully to $150 \mathrm{~mm}$ diameter billets. Work in progress is aimed at further scale-up of multifilamentary extrusion billets to $250-300 \mathrm{~mm}$ diameter billets.

3. Successful magnet tests of this material have confirmed that long lengths can be fabricated with uniform properties and good process control.

\section{Acknowledgments}

We acknowledge the contributions of the staff at Supercon, Inc. for their dedication and perseverance in producing this APC material for our evaluation. We also acknowledge the help of Bill Sampson and his group at BNL in measuring the cable critical currents. Finally, we acknowledge the contributions of the technical staff at LBL in construction of the magnets and the analysis of the APC samples.

\section{Refierences}

[1] P. J. Lee and D. C. Larbalestier, "An Examination of the Properties of SSC Phase II R\&D Strands", IEEE Trans. on Applied Superconductivity 3,833-841, 1993.

[2] R. M. Scanlan, "The Evolution of Tooling, Techniques, and Quality Control for Accelerator Dipole Magnet Cables", Ibid. pp 842-849.

[3] L.D. Cooley, P.D. Jablonski, P.J. Lee, and D. C. Larbalestier, "Strongly Enhanced Critical Current Density in Nb47wt\% Ti Having a Highly Aligned Microstructure", App. Phys. LetL. 58, 2984-2986, 1991.

[4] G. L. Dorofejev et al. "Artificial Pinning Centers", Proc. 9th Conf. on Magnet Technology, C. Marinucci and P. Weymouth eds., Swiss Inst. for Nucl. Res., 564-566, 1985.

[5] H.C. Kanithi, P. Valaris, L.R. Motowidlo, B.A. Zeitlin, and R. M. Scanlan, "Further Developments in $\mathrm{NbTi}$ Superoonductors with Artificial Pinning Centers", Adv. in Cryo. Eng. (Materials), 38, 675683, 1992.

[6] U.S. Patents 5,160,550, 5,160,794 and 1,158,620.

[7] P. J. Lee, J. C. McKinnell, and D. C. Larbalestier, "Restricted, Novel Heat Treatments for Obtaining High Jc in Nb46wt\% Ti", Adv. in Cryo. Eng. (Materials), 36, 287-294, 1990.

[8] R. M. Scanlan, D. R. Dietderich, W. Ghiorso and P. Mc Manaman, "Characterization and Coil Test Results for a Multifilamantary NbTi Conductor Utilizing Artificial Pinning Center Technology", IEEE Trans. on Appl. Superconductivity, 3, 1358-1361, 1993.

[9] J. M. Seuntjens, F. Y. Clark, T.J. Headley, F.A. Geulich, and N.Y.C. Yang, "Image Analysis of Superconducting Composites", Ibid., pp.729-733.

[10] D. Dell'Orco, S. Caspi, J. O'Neill, A. Lietzke, R. Scanlan, C.E. Taylor and A. Wandesforde, "A $50 \mathrm{~mm}$ Bore Superconducting Dipole with a unique Iron Yoke Structure", IBid., pp. 637-641.

[11] M. Garber, A.K. Ghosh and W. B. Sampson, "The Effect of Self Field on the Critical Current Determination of Multifilamentary superconudctors", Proc. IEEE Trans. on Magnetics, MAG-25, 19401944, 1989.

[12] W.B. Sampson, private communication.

[13] P.A. Sanger, private communications.

[14] W.K. McDonald, "Fabrication of Niobium and Niobium Alloys". Proc. of Int. Symp. Niobium 81. H. Stuar, Ed., 225-238. 1984. 

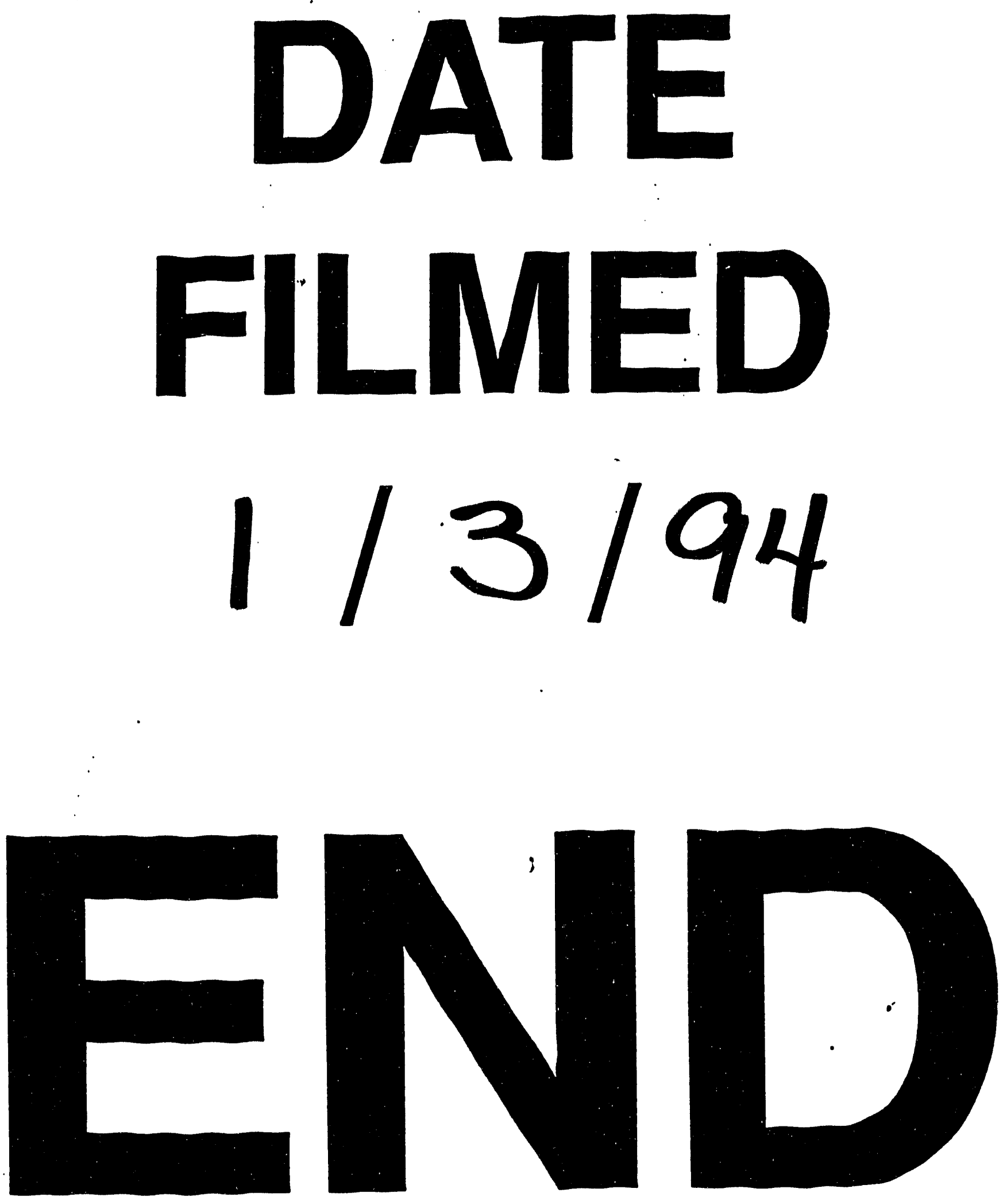
